\title{
Gaucher Disease
}

National Institute of Neurological Disorders and Stroke (NINDS)

\section{Source}

National Institute of Neurological Disorders and Stroke (NINDS). Gaucher Disease Information Page.

Gaucher disease is one of the inherited metabolic disorders known as lipid storage diseases. Lipids are fatty materials that include oils, fatty acids, waxes, and steroids (such as cholesterol and estrogen). People with Gaucher disease either do not produce enough of the enzyme glucocerebrosidase needed to break down lipids or have enzymes that do not work properly. Fatty materials can accumulate in the brain and other organs. General symptoms may beg in in early life or adulthood and include skeletal disorders and bone lesions that may cause pain and fractures, enlarged spleen and liver, liver malfunction, anemia, and yellow spots in the eyes.

There are three common clinical subtypes of Gaucher disease:

- Type 1 (or nonneuropathic) typically does not affect the brain. Symptoms may beg in early in life or in adulthood. People in this group usually bruise easily due to low blood platelets and experience fatigue due to anemia. They also may have an enlarged liver and spleen. Many individuals with a mild form of the disorder may not show any symptoms.

- Type 2 Gaucher disease (acute infantile neuropathic Gaucher disease) symptoms usually beg in by 3 months of age and includes extensive brain damage, seizures, spasticity, poor ability to suck and swallow, and enlarged liver and spleen. Affected children usually die before 2 years of age.

- Type 3 (or chronic neuropathic Gaucher disease) includes signs of brain involvement, seizures, skeletal irregularities, eye movement disorders, cognitive deficit, poor coordination, enlarged liver and spleen, respiratory problems, and blood disorders. 